\title{
A general inexact iterative method for monotone operators, equilibrium problems and fixed point problems of semigroups in Hilbert spaces
}

\author{
Vittorio Colao ${ }^{1 *}$, Giuseppe Marino ${ }^{1}$ and Daya Ram Sahu ${ }^{2}$
}

\author{
* Correspondence: colao@mat. \\ unical.it \\ 'Dipartimento di Matematica, \\ Universita della Calabria, Arcavacata \\ di Rende (Cs) 87036, Italy \\ Full list of author information is \\ available at the end of the article
}

\begin{abstract}
Let $H$ be a real Hilbert space. Consider on $H$ a nonexpansive family $\mathcal{T}=\{T(t): 0 \leq t<\infty\}$ with a common fixed point, a contraction $f$ with the coefficient $0<\alpha<1$, and a strongly positive linear bounded self-adjoint operator $A$ with the coefficient $\bar{\gamma}>0$. Assume that $0<\gamma<\bar{\gamma} / \alpha$ and that $\mathcal{S}=\{S(t): 0 \leq t<\infty\}$ is a family of nonexpansive self-mappings on $H$ such that $F(\mathcal{T}) \subseteq F(\mathcal{S})$ and $\mathcal{T}$ has property $(\mathcal{A})$ with respect to the family $\mathcal{S}$. It is proved that the following schemes (one implicit and one inexact explicit):

$$
x_{t}=b_{t} \gamma f\left(x_{t}\right)+\left(I-b_{t} A\right) S(t) x_{t}
$$

and

$$
x_{0} \in H, \quad x_{n+1}=\alpha_{n} \gamma f\left(x_{n}\right)+\beta_{n} x_{n}+\left(\left(1-\beta_{n}\right) I-\alpha_{n} A\right) S\left(t_{n}\right) x_{n}+e_{n}, \quad n \geq 0
$$

converge strongly to a common fixed point $x^{*} \in F(\mathcal{T})$, where $F(\mathcal{T})$ denotes the set of common fixed point of the nonexpansive semigroup. The point $x^{*}$ solves the variational in-equality $\left\langle(\gamma f-A) x^{*}, x-x^{*}\right\rangle \leq 0$ for all $x \in F(\mathcal{T})$. Various applications to zeros of monotone operators, solutions of equilibrium problems, common fixed point problems of nonexpansive semigroup are also presented. The results presented in this article mainly improve the corresponding ones announced by many others.

2010 Mathematics Subject Classification: 47H09; 47J25.
\end{abstract}

Keywords: nonexpansive semigroup, common fixed point, contraction, variational inequality

\section{Introduction}

Let $H$ be a real Hilbert space and $T$ be a nonlinear mapping with the domain $D(T)$. A point $x \in D(T)$ is a fixed point of $T$ provided $T x=x$. Denote by $F(T)$ the set of fixed points of $T$; that is, $F(T)=\{x \in D(T): T x=x\}$. Recall that $T$ is said to be nonexpansive if

$$
\|T x-T y\| \leq\|x-y\|, \forall x, y \in D(T)
$$

Recall that a family $\mathcal{T}=\{T(s): s \geq 0\}$ of mappings from $H$ into itself is called a oneparameter nonexpansive semigroup if it satisfies the following conditions:

(i) $T(0) x=x, \forall x \in H$;

(ii) $T(s+t) x=T(s) T(t) x, \forall s, t \geq 0$ and $\forall x \in H$;

(c) 2012 Colao et al; licensee Springer. This is an Open Access article distributed under the terms of the Creative Commons Attribution License (http://creativecommons.org/licenses/by/2.0), which permits unrestricted use, distribution, and reproduction in any medium, provided the original work is properly cited. 
(iii) $\|T(s) x-T(s) y\| \leq\|x-y\|, \forall s \geq 0$ and $\forall x, y \in H$;

(iv) for all $x \in C, s \mapsto T(s) x$ is continuous.

We denote by $F(\mathcal{T})$ the set of common fixed points of $\mathcal{T}$, that is, $F(\mathcal{T})=\cap_{0 \leq s \leq \infty} F(T(s))$. For each $t>0$ and $x \in C, \sigma_{t}(x)$ is the average defined by $\sigma_{t}(x)=\frac{1}{t} \int_{0}^{t} T(s) x d s$. It is known that $F(\mathcal{T})$ is closed and convex; see [1]. Let $C$ be a nonempty closed and convex subset of $H$. One classical way to study nonexpansive mappings is to use contractions to approximate a nonexpansive mapping; see [2,3]. More precisely, take $t \in(0,1)$ and define a contraction $T_{t}: C \rightarrow C$ by

$$
T_{t} x=t u+(1-t) T x, \quad x \in C,
$$

where $u \in C$ is a fixed element. Banach's contraction mapping principle guarantees that $T_{t}$ has a unique fixed point $x_{t}$ in $C$. It is unclear, in general, what the behavior of $\left\{x_{t}\right\}$ is as $t \rightarrow 0$, even $T$ has a fixed point. However, in the case of $T$ having a fixed point, Browder [2] proved the following well-known strong convergence theorem.

Theorem B. Let $C$ be a closed convex bounded subset of a Hilbert space $H$ and let $T$ be a nonexpansive mapping on $C$. Fix $u \in C$ and define $z_{t} \in C$ as $z_{t}=t u+(1-t) T z_{t}$ for $t \in(0,1)$. Then as $t \rightarrow 0,\left\{z_{t}\right\}$ converges strongly to an element of $F(T)$ nearest to $u$.

As motivated by Theorem B, Halpern [4] considered the following explicit iteration:

$$
x_{0} \in C, \quad x_{n+1}=\alpha_{n} u+\left(1-\alpha_{n}\right) T x_{n}, \quad n \geq 0,
$$

and proved the following theorem.

Theorem H. Let $C$ be a closed convex bounded subset of a Hilbert space $H$ and let $T$ be a nonexpansive mapping on C. Define a real sequence $\left\{\alpha_{n}\right\}$ in $[0,1]$ by $\alpha_{n}=n^{-\theta}$, $0<\theta<1$. Define a sequence $\left\{x_{n}\right\}$ by (1.2). Then $\left\{x_{n}\right\}$ converges strongly to the element of $F(T)$ nearest to $u$.

In 1977, Lions [5] improved the result of Halpern, still in Hilbert spaces, by proving the strong convergence of $\left\{x_{n}\right\}$ to a fixed point of $T$ where the real sequence $\left\{\alpha_{n}\right\}$ satisfies the following conditions:

(C1) $\lim _{n \rightarrow \infty} \alpha_{n}=0$;

(C2) $\sum_{n=1}^{\infty} \alpha_{n}=\infty$;

(C3) $\lim _{n \rightarrow \infty} \frac{\alpha_{n+1}-\alpha_{n}}{\alpha_{n+1}^{2}}=0$.

It was observed that both Halpern's and Lions's conditions on the real sequence $\left\{\alpha_{n}\right\}$ excluded the canonical choice $\alpha_{n}=\frac{1}{n+1}$. This was overcome in 1992 by Wittmann [6], who proved, still in Hilbert spaces, the strong convergence of $\left\{x_{n}\right\}$ to a fixed point of $T$ if $\left\{\alpha_{n}\right\}$ satisfies the following conditions:

(C1) $\lim _{n \rightarrow \infty} \alpha_{n}=0$;

(C2) $\sum_{n=1}^{\infty} \alpha_{n}=\infty$;

(C4) $\sum_{n=1}^{\infty}\left|\alpha_{n+1}-\alpha_{n}\right|<\infty$.

Recall that a mapping $f: H \rightarrow H$ is an $\alpha$-contraction if there exists a constant $\alpha \in(0,1)$ such that

$$
\|f(x)-f(y)\| \leq \alpha\|x-y\|, \quad \forall x, y \in H .
$$

Recall that an operator $A$ is strongly positive on $H$ if there exists a constant $\bar{\gamma}>0$ such that 


$$
\langle A x, x\rangle \geq \bar{\gamma}\|x\|^{2}, \quad \forall x \in H
$$

Iterative methods for nonexpansive mappings have recently been applied to solve convex minimization problems; see, e.g., [7-13] and the references therein. A typical problem is to minimize a quadratic function over the set of the fixed points of a nonexpansive mapping on a real Hilbert space $H$ :

$$
\min _{x \in D} \frac{1}{2}\langle A x, x\rangle-\langle x, b\rangle
$$

where $A$ is a linear bounded operator, $D$ is the fixed point set of a nonexpansive mapping $T$ and $b$ is a given point in $H$. In [11], it is proved that the sequence $\left\{x_{n}\right\}$ defined by the iterative method below, with the initial guess $x_{0} \in H$ chosen arbitrarily,

$$
x_{n+1}=\left(I-\alpha_{n} A\right) T x_{n}+\alpha_{n} b, \quad n \geq 0,
$$

strongly converges to the unique solution of the minimization problem (1.2) provided the sequence $\left\{\alpha_{n}\right\}$ satisfies certain conditions.

Marino and $\mathrm{Xu}[10]$ studied the following continuous scheme

$$
x_{t}=t \gamma f\left(x_{t}\right)+(I-t A) T x_{t},
$$

where $f$ is an $\alpha$-contraction on a real Hilbert space $H, A$ is a bounded linear strongly positive operator and $\gamma>0$ is a constant. They showed that $\left\{x_{t}\right\}$ strongly converges to a fixed point $\bar{x}$ of $T$. Also in [10] they introduced a general explicit iterative scheme by the viscosity approximation method:

$$
x_{n} \in H, \quad x_{n+1}=\alpha_{n} \gamma f\left(x_{n}\right)+\left(I-\alpha_{n} A\right) T x_{n}, \quad n \geq 0
$$

and proved that the sequence $\left\{x_{n}\right\}$ generated by (1.3) converges strongly to a unique solution of the variational inequality:

$$
\left\langle(A-\gamma f) x^{*}, x-x^{*}\right\rangle \geq 0, \quad \forall x \in F(T),
$$

which is the optimality condition for the minimization problem:

$$
\min _{x \in F(T)} \frac{1}{2}\langle A x, x\rangle-h(x)
$$

where $h$ is a potential function for $\gamma f$ (i.e., $h^{\prime}(x)=\gamma f(x)$ for $x \in H$ ).

It is an interesting problem to study above (Browder's, Halpern's and so on) results with respect to the nonexpansive semigroup case. So far, only partial answers have been obtained. Recently, Plubtieng and Punpaeng [14] considered the iteration process $\left\{x_{n}\right\}$ generated by

$$
x_{0} \in H, x_{n+1}=\alpha_{n} f\left(x_{n}\right)+\beta_{n} x_{n}+\left(1-\alpha_{n}-\beta_{n}\right) \frac{1}{s_{n}} \int_{0}^{s_{n}} T(s) x_{n} d s, n \geq 0,
$$

where $\left\{\alpha_{n}\right\},\left\{\beta_{n}\right\} \subset(0,1)$ with $\alpha_{n}+\beta_{n}<1$ and $\left\{t_{n}\right\}$ is a positive real divergent sequence. They proved, under certain appropriate conditions on $\left\{\alpha_{n}\right\}$, that $\left\{x_{n}\right\}$ converges strongly to a common fixed point of one-parameter nonexpansive semigroup $\mathcal{T}=\{T(s): s \geq 0\}$. 
In this article, motivated by $\mathrm{Li}$ et al. [8], Marino and $\mathrm{Xu}$ [10], Plubtieng and Punpaeng [14], Cianciaruso et al. [15], Shioji and Takahashi [16] and Shimizu and Takahashi [17], we consider the following more general schemes (one implicit and one inexact explicit):

$$
x_{t}=b_{t} \gamma f\left(x_{t}\right)+\left(I-b_{t} A\right) S(t) x_{t}
$$

and

$$
x_{0} \in H, \quad x_{n+1}=\alpha_{n} \gamma f\left(x_{n}\right)+\beta_{n} x_{n}+\left(\left(1-\beta_{n}\right) I-\alpha_{n} A\right) S\left(t_{n}\right) x_{n}+e_{n}, \quad n \geq 0
$$

where $\mathcal{T}=\{T(t): 0 \leq t<\infty\}$ is a family of arbitrary nonexpansive self-mappings on $H$ with a common fixed point, $\mathcal{S}=\{S(t): 0 \leq t<\infty\}$ is a family of nonexpansive selfmappings on $H$ such that $\mathcal{T}$ has property $(\mathcal{A})$ with respect to the family $\mathcal{S}$ and $F(\mathcal{T}) \subseteq F(\mathcal{S}), \gamma>0$ is a constant, $f: H \rightarrow H$ is an $\alpha$-contraction, $A$ is a bounded linear strongly positive self-adjoint operator on $H$ and $\left\{b_{t}\right\}$ is a net in $(0,1)$. Furthermore, by applying these results, we obtain iterative algorithms for zeros of monotone operators, equilibrium problems, and common fixed point problems of nonexpansive semigroups in real Hilbert spaces.

The results presented in this article improve and extend the corresponding results announced by Marino and Xu [10], Plubtieng and Punpaeng [14], Cianciaruso et al. [15], Shioji and Takahashi [16], and Shimizu and Takahashi [17]. We remark that our results are very similar to those of $\mathrm{Li}$ et al. [8]. However, it seems that can be a gap in the proofs of $\mathrm{Li}$ et al. results. Indeed, their semigroups and the contraction are selfmappings defined on a closed convex subset $\mathrm{C}$ of the Hilbert space $H$, while the strongly positive linear bounded operator is defined on $H$. So both the schemes involve not a convex combination, that this they are of interest only in the case $C=H$.

\section{Preliminaries}

This section collects some lemmas which will be used in the proofs for the main results in the following section. Some of them are known; others are not hard to derive.

Lemma 2.1. (Shimizu and Takahashi [[17], Lemma 2]). Let $C$ be a nonempty closed convex bounded subset of a Hilbert space $H$, and $\mathcal{T}=\left\{T(t): t \in \mathbb{R}^{+}\right\}$a strongly continuous semigroup of nonexpansive mappings from $C$ into itself. Let $\sigma_{t}(x):=\frac{1}{t} \int_{0}^{t} T(s) x d s$. Then

$$
\lim _{t \rightarrow \infty} \sup _{x \in D}\left\|\sigma_{t}(x)-T(h) \sigma_{t}(x)\right\|=0 \text { for all } h>0 .
$$

Lemma 2.2. ([[18], Corollary 5.6.4], [19]) (Demiclosedness principle) Let $\mathrm{H}$ be a Hilbert space, $C$ is a closed convex subset of $H$, and $T: C \rightarrow C$ a nonexpansive mapping. Then $I-T$ is demiclosed, i.e., if $\left\{x_{n}\right\}$ is a sequence in $C$ weakly converging to $x$ and if $\left\{(I-T) x_{n}\right\}$ strongly converges to $y$, then $(I-T) x=y$.

Lemma 2.3. ([[18], Corollary 5.2.29]) Let $C$ be a nonempty closed convex subset of a strictly convex Banach space $X$ and $T: C \rightarrow C$ a nonexpansive mapping. Then $F(T)$ is closed and convex.

Lemma 2.4. Let $C$ be a nonempty closed convex subset of a real Hilbert space $H$ and let $P_{C}$ be the metric projection from $H$ onto $C$ (i.e., for $x \in H, P_{C} x$ is the only point in $C$ 
such that $\left.\left\|x-P_{C} x\right\|=\inf \{\|x-z\|: z \in C\}\right)$. Given $x \in H$ and $z \in C$. Then $z=P_{C} x$ if and only if there holds the relations:

$$
\langle x-z, y-z\rangle \leq 0, \forall y \in C .
$$

Lemma 2.5. Let $H$ be a real Hilbert space, $f: H \rightarrow H$ an $\alpha$-contraction, and $A$ is a strongly positive linear bounded self-adjoint operator on $H$ with the coefficient $\bar{\gamma}>0$. Then, for $0<\gamma<\bar{\gamma} / \alpha$,

$$
\langle x-y,(A-\gamma f) x-(A-\gamma f) y\rangle \geq(\bar{\gamma}-\gamma \alpha)\|x-y\|^{2}, \quad x, y \in H .
$$

That is, $A-\gamma$ is strongly monotone with coefficient $\bar{\gamma}-\alpha \gamma$.

Remark 2.6. Taking $\gamma=1$ and $A=I$, the identity mapping, we have the following inequality:

$$
\langle x-y,(I-f) x-(I-f) y\rangle \geq(1-\alpha)\|x-y\|^{2}, \quad x, y \in H .
$$

Furthermore, if $f$ is a nonexpansive mapping in Remark 2.6, we have

$$
\langle x-y,(I-f) x-(I-f) y\rangle \geq 0, \quad x, y \in H .
$$

Lemma 2.7.[10]. Assume $A$ is a strongly positive linear bounded self-adjoint operator on a real Hilbert space $H$ with coefficient $\bar{\gamma}>0$ and $0<\rho \leq\|A\|^{-1}$. Then $\|I-\rho A\| \leq 1-\rho \bar{\gamma}$.

Lemma 2.8. [12]. Let $\left\{\alpha_{n}\right\}$ be a sequence of nonnegative real numbers satisfying the following condition:

$$
\alpha_{n+1} \leq\left(1-\gamma_{n}\right) \alpha_{n}+\gamma_{n} \sigma_{n}, \quad \forall n \geq 0,
$$

where $\left\{\gamma_{n}\right\}$ is a sequence in $(0,1)$ and $\left\{\sigma_{n}\right\}$ is a sequence of real numbers such that

(i) $\lim _{n \rightarrow \infty} \gamma_{n}=0$ and $\sum_{n=0}^{\infty} \gamma_{n}=\infty$,

(ii) either $\lim \sup _{n \rightarrow \infty} \sigma_{n} \leq 0$ or $\sum_{n=0}^{\infty}\left|\gamma_{n} \sigma_{n}\right|<\infty$.

Then $\left\{\alpha_{n}\right\}_{n=0}^{\infty}$ converges to zero.

Let $C$ be a nonempty subset of a Banach space $X$. Throughout this article, $G$ denotes an unbounded set of $\mathbb{R}^{+}:=[0, \infty)$ such that $s+t \in G$ for all $s, t \in G$ (often $G=\mathbb{N o r} \mathbb{R}^{+}$) and $\mathcal{B}(\mathrm{C})$ denotes collection of all bounded subsets of $C$. Let $\mathcal{T}=\left\{T_{s}: s \in G\right\}$ be a family of mappings from $C$ into itself. Then:

(i) a sequence $\left\{x_{n}\right\}$ in $C$ is said to be an approximate fixed point sequence of $\mathcal{T}$ if $\lim _{n \rightarrow \infty}\left\|x_{n}-T_{\mathcal{T}} x_{n}\right\|=0$ for all $\tau \in G$,

(ii) $\mathcal{T}=\left\{T_{s}: s \in G\right\}$ is said to uniformly asymptotically regular on $C$ (for short, u.a.r. on $C$ ) (see, [20]) if

$$
\lim _{t \in G, t \rightarrow \infty}\left(\sup _{x \in \tilde{C}}\left\|T_{t} x-T_{s} T_{t} x\right\|\right)=0 \text { for all } s \in G \text { and } \tilde{C} \in \mathcal{B}(C) .
$$

A family $\mathcal{T}=\left\{T_{s}: s \in G\right\}$ satisfies property $(\mathcal{A})$ if the following holds: each $\left\{x_{s}\right\}_{s \in G} \in \mathcal{B}(C)$ with $x_{s}-T_{s} x_{s} \rightarrow 0$ as $s \rightarrow \infty \Rightarrow x_{s}-T_{t} x_{s} \rightarrow \infty$ for all $t \in G$.

Remark 2.9. If $\mathcal{T}$ be a singleton, i.e., $\mathcal{T}=\{T\}$, or $T_{s}=T$ for all $s$ in $G$, then $\{T\}$ always has property $(\mathcal{A})$.

We further remark that the notion of uniform asymptotic regularity introduced by Edelstein and O'Brien [21] plays an important role for studying property $(\mathcal{A})$ of 
nonlinear Lipschitzian-type operators. Indeed, if $\mathcal{T}=\left\{T_{s}: s \in G\right\}$ is a nonexpansive semigroup and u.a.r., then $\mathcal{T}$ has property $(\mathcal{A})$. Indeed, for $\left\{\gamma_{s}\right\} \in \mathcal{B}(C)$ and $t \in G$,

$$
\begin{aligned}
\left\|y_{s}-T_{t} y_{s}\right\| & \leq\left\|y_{s}-T_{s} y_{s}\right\|+\left\|T_{s} y_{s}-T_{t} T_{s} y_{s}\right\|+\left\|T_{t} T_{s} y_{s}-T_{t} y_{s}\right\| \\
& \leq 2\left\|y_{s}-T_{s} y_{s}\right\|+\sup _{y \in\left\{y_{\gamma}: \gamma \in G\right\}}\left\|T_{s} y-T_{t} T_{s} y\right\| \rightarrow 0 \text { as } s \rightarrow \infty .
\end{aligned}
$$

We now introduce property $(\mathcal{A})$ of $\mathcal{T}$ with respect to the family $\mathcal{G}$.

Let $C$ be a nonempty subset of a Banach space $X$ and $\mathcal{T}=\left\{T(t): t \in \mathbb{R}^{+}\right\}$be a family of mappings from $C$ into itself with $\cap_{t>0} F(T(t)) \neq \varnothing$. Let $\mathcal{G}=\left\{G_{t}: t \in \mathbb{R}^{+}\right\}$be a family of mappings from $C$ into itself such that $n_{t>0} F(T(t)) \neq n_{t>0} F\left(G_{t}\right)$. We say the family $\mathcal{T}=\{T(s): s \in G\}$ has property $(\mathcal{A})$ with respect to the family $\mathcal{G}=\left\{G_{t}: t \in \mathbb{R}^{+}\right\}$if the following holds:

each $\left\{x_{s}\right\}_{s \in G} \in \mathcal{B}(C)$ with $x_{s}-G_{s} x_{s} \rightarrow 0$ as $s \rightarrow \infty \Rightarrow x_{s}-T(t) x_{s} \rightarrow 0$ as $s \rightarrow \infty$ for all $t>0$.

Remark 2.10. If a family $\mathcal{T}=\{T(s): s \in G\}$ has property $(\mathcal{A})$, then $\mathcal{T}$ has property $(\mathcal{A})$ with respect to itself.

We now give some examples.

Example 2.11. Let $C$ be a nonempty closed convex subset of a Banach space $X$ and $T$ be a nonexpansive mapping from $C$ into itself with $F(T) \neq \varnothing$. For each $t \in G$, and $b_{t} \in \mathbb{R}$ with $0<a \leq b_{t} \leq b<1$, define $G_{t}: C \rightarrow C$ by

$$
G_{t} x=\left(1-b_{t}\right) x+b_{t} T x \text { for all } x \in C .
$$

Then $T$ has property $(\mathcal{A})$ with respect to family $\left\{G_{t}: t \in G\right\}$.

Proof. Let $\left\{x_{t}\right\}_{t \in G} \in \mathcal{B}(C)$ such that $\left\|x_{t}-G_{t}\left(x_{t}\right)\right\| \rightarrow 0$ as $t \rightarrow \infty$. Note that

$$
\left\|x_{t}-T x_{t}\right\|=b_{t}\left\|x_{t}-G_{t}\left(x_{t}\right)\right\|
$$

and $0<a \leq b_{t} \leq b<1$ for all $t \in G$. Therefore, $\left\|x_{t}-T x_{t}\right\| \rightarrow 0$ as $t \rightarrow \infty$. $\square$

The following proposition shows that in a uniformly convex Banach space, nonexpansive semigroup $\mathcal{T}=\left\{T(t): t \in \mathbb{R}^{+}\right\}$has property $(\mathcal{A})$ with respect to a nonexpansive semigroup $\left\{\sigma_{t}: t \in \mathbb{R}^{+}\right\}=\left\{\frac{1}{t} \int_{0}^{t} T(s) x d s: t \in \mathbb{R}^{+}\right\}$.

Example 2.12. Let D be a nonempty closed convex bounded subset of a Hilbert space $H$, and $\mathcal{T}=\left\{T(t): t \in \mathbb{R}^{+}\right\}$be a strongly continuous semigroup of nonexpansive mappings from $D$ into itself. For each $t>0$, let $x_{t} \in D$ such that $\left\|x_{t}-\sigma_{t}\left(x_{t}\right)\right\| \rightarrow 0$ as $t \rightarrow \infty$. Then $\left\|x_{t}-T(\mathcal{T}) x_{t} \rightarrow 0\right\|$ as $t \rightarrow \infty$ for each $\tau>0$.

Proof. Let $\tau>0$. Observe that

$$
\begin{aligned}
\left\|T(\tau) x_{t}-x_{t}\right\| & \leq\left\|T(\tau) x_{t}-T(\tau) \sigma_{t}\left(x_{t}\right)\right\|+\left\|T(\tau) \sigma_{t}\left(x_{t}\right)-\sigma_{t}\left(x_{t}\right)\right\|+\left\|\sigma_{t}\left(x_{t}\right)-x_{t}\right\| \\
& \leq 2\left\|x_{t}-\sigma_{t}\left(x_{t}\right)\right\|+\left\|T(\tau) \sigma_{t}\left(x_{t}\right)-\sigma_{t}\left(x_{t}\right)\right\| \\
& \leq 2\left\|x_{t}-\sigma_{t}\left(x_{t}\right)\right\|+\sup _{x \in D}\left\|T(\tau) \sigma_{t}(x)-\sigma_{t}(x)\right\| .
\end{aligned}
$$

By Lemma 2.1, we obtain that $\left\|x_{t}-T(\tau) x_{t}\right\| \rightarrow 0$ as $t \rightarrow \infty$ for each $\tau>0$. $\square$

\section{Main results}

Let $H$ be a real Hilbert space and $\mathcal{S}=\{S(t): 0 \leq t<\infty\}$ a family of nonexpansive selfmappings on $H$ with $F(\mathcal{S}) \neq \emptyset$. By Lemma $2.3, F(\mathcal{S})$ is closed and convex. Let $A$ be a strongly positive linear bounded self-adjoint operator of $H$ into itself with coefficient $\bar{\gamma}>0$ and $f: H \rightarrow H$ an $\alpha$-contraction. Assume that $0<\gamma<\bar{\gamma} / \alpha$ and $\left\{b_{t}: t>0\right\}$ is 
a net in $\left(0,\|A\|^{-1}\right)$ such that $\lim _{t \rightarrow \infty} b_{t}=0$. For each $t>0$, the mapping $G_{t}: H \rightarrow H$ defined by

$$
G_{t} x:=b_{t} \gamma f(x)+\left(I-b_{t} A\right) S(t) x, \quad x \in H
$$

is a contraction with Lipschitz constant $1-b_{t}(\bar{\gamma}-\alpha \gamma)$. Indeed, for all $x, y \in H$, we have

$$
\begin{aligned}
\left\|G_{t} x-G_{t} y\right\| & \leq\left\|\left(1-b_{t} A\right)(S(t) x-S(t) \gamma)\right\|+\gamma b_{t}\|f x-f y\| \\
& \leq\left(1-b_{t} \bar{\gamma}\right)\|x-\gamma\|+\gamma b_{t} \alpha\|x-\gamma\| \\
& =\left[1-b_{t}(\bar{\gamma}-\alpha \gamma)\right]\|x-\gamma\| .
\end{aligned}
$$

By the Banach contraction principle, $G_{t}$ has a unique fixed point, denoted by, $x_{t}$ in $H$, which uniquely solves the fixed point equation

$$
x_{t}=b_{t} \gamma f\left(x_{t}\right)+\left(I-b_{t} A\right) S(t) x_{t} .
$$

Lemma 3.1. Let $H$ be a real Hilbert space and $\mathcal{S}=\{S(t): 0 \leq t<\infty\}$ be a family of nonexpansive self-mappings on $H$ such that $F(\mathcal{S}) \neq \emptyset$. Let $f: H \rightarrow H$ be an $\alpha$-contraction, $A$ is a strongly positive linear bounded self-adjoint operator of $H$ into itself with coefficient $\bar{\gamma}>0$. Let $\left\{b_{t}: t>0\right\}$ be a net in $\left(0,\|A\|^{-1}\right)$ such that $\lim _{t \rightarrow \infty} b_{t}=0$. Assume that $0<\gamma<\bar{\gamma} / \alpha$ and $x_{t}$ is defined by (3.1). Then we have the following:

(a) There is a nonempty closed convex bounded subset $D$ of $H$ such that $D$ is $S(t)$ invariant for each $t>0$ and $\left\{x_{t}\right\}$ is in $D$.

(b) $\left\|x_{t}-S(t) x_{t}\right\| \rightarrow 0$ as $t \rightarrow \infty$.

Proof. (a) Taking $p \in F(\mathcal{S})$, we have

$$
\begin{aligned}
\left\|x_{t}-p\right\| & =\left\|b_{t} \gamma f\left(x_{t}\right)+\left(I-b_{t} A\right) S(t) x_{t}-p\right\| \\
& \leq b_{t}\left\|\gamma f\left(x_{t}\right)-A p\right\|+\left(1-b_{t} \bar{\gamma}\right)\left\|S(t) x_{t}-p\right\| \\
& \leq b_{t}\left\|\gamma f\left(x_{t}\right)-A p\right\|+\left(1-b_{t} \bar{\gamma}\right)\left\|x_{t}-p\right\| \\
& \leq b_{t} \gamma\left\|f\left(x_{t}\right)-f(p)\right\|+b_{t}\|\gamma f(p)-A p\|+\left(1-b_{t} \bar{\gamma}\right)\left\|x_{t}-p\right\| \\
& \leq\left[1-b_{t}(\bar{\gamma}-\gamma \alpha)\right]\left\|x_{t}-p\right\|+b_{t}\|\gamma f(p)-A p\| .
\end{aligned}
$$

It follows that

$$
\left\|x_{t}-p\right\| \leq \frac{1}{\bar{\gamma}-\alpha \gamma}\|\gamma f(p)-A p\| .
$$

This implies that $\left\{x_{t}\right\}$ is bounded. Let $D$ be the ball $B(p, r)$, centered in $p$ and with radius $r=\frac{1}{\bar{\gamma}-\alpha \gamma}\|\gamma f(p)-A p\|, \quad$ i.e., $\quad D=\left\{w \in H:\|w-p\| \leq \frac{1}{\bar{\gamma}-\alpha \gamma}\|\gamma f(p)-A p\|\right\}$. Then $\left\{x_{t}\right\}$ is contained in set $D$. Moreover,

$$
\begin{aligned}
\left\|S(t) x_{t}-p\right\| & =\left\|S(t) x_{t}-S(t) p\right\| \\
& \leq\left\|x_{t}-p\right\| \\
& \leq \frac{1}{\bar{\gamma}-\gamma \alpha}\|\gamma f(p)-A p\| .
\end{aligned}
$$

Thus, $D$ is a nonempty closed convex bounded subset of $H$ and $S(t)$-invariant.

(b) The boundedness of $\left\{x_{t}\right\}$ implies that $\left\{x_{t}\right\}$ and $\left\{A S(t) x_{t}\right\}$ are bounded. Thus, $\left\|x_{t}-S(t) x_{t}\right\|=b_{t}\left\|\gamma f\left(x_{t}\right)-A S(t) x_{t}\right\| \rightarrow 0$ as $t \rightarrow \infty$.

We now establish our strong convergence theorems.

Theorem 3.2. (Implicit scheme) Let $H$ be a real Hilbert space $H$ and $\mathcal{T}=\{T(t): 0 \leq t<\infty\}$ be a family of nonexpansive self-mappings on $H$ such that 
$F(\mathcal{T}) \neq \emptyset$. Let $f: H \rightarrow H$ be an $\alpha$-contraction and $A$ be a strongly positive linear bounded self-adjoint operator on $H$ with the coefficient $\bar{\gamma}>0$. Let $\mathcal{S}=\{S(t): 0 \leq t<\infty\}$ be a family of nonexpansive self-mappings on $H$ such that $\mathcal{T}$ has property $(\mathcal{A})$ with respect to the family $\mathcal{S}$ and $F(\mathcal{T}) \subseteq F(\mathcal{S})$. Assume that $0<\gamma<\bar{\gamma} / \alpha$ and that $\left\{b_{t}: t>0\right\}$ is a net in $\left(0,\|A\|^{-1}\right)$ such that $\lim _{t \rightarrow \infty} b_{t}=0$. Then $\left\{x_{t}\right\}$ defined by (3.1) strongly converges as $t \rightarrow \infty$ to $x^{*} \in F(\mathcal{T})$, where $x^{*}=P_{F(\mathcal{T})}(I-A+\gamma f)$ is a solution of the following variational inequality

$$
\left\langle(\gamma f-A) x^{*}, p-x^{*}\right\rangle \leq 0, \quad \forall p \in F(\mathcal{T}) .
$$

Proof. The uniqueness of the solution of the variational inequality (3.2) is a consequence of the strong monotonicity of $A-\gamma f$ (Lemma 2.5). Next, we shall use $x^{*} \in F(\mathcal{T})$ to denote the unique solution of (3.2). To prove that $x_{t} \rightarrow x^{\prime \prime}(t \rightarrow \infty)$, we write, for a given $p \in F(\mathcal{T})$,

$$
x_{t}-p=b_{t}\left(\gamma f\left(x_{t}\right)-A p\right)+\left(I-b_{t} A\right)\left(S(t) x_{t}-p\right) .
$$

Using $x_{t}-p$ to make inner product, we obtain that

$$
\begin{aligned}
\left\|x_{t}-p\right\|^{2} & =\left\langle\left(I-b_{t} A\right)\left(S(t) x_{t}-p\right), x_{t}-p\right\rangle+b_{t}\left\langle\gamma f\left(x_{t}\right)-A p, x_{t}-p\right\rangle \\
& \leq\left(1-b_{t} \bar{\gamma}\right)\left\|x_{t}-p\right\|^{2}+b_{t}\left\langle\gamma f\left(x_{t}\right)-A p, x_{t}-p\right\rangle .
\end{aligned}
$$

It follows that

$$
\begin{aligned}
\left\|x_{t}-p\right\|^{2} & \leq \frac{1}{\bar{\gamma}}\left(\gamma\left\langle f\left(x_{t}\right)-f(p), x_{t}-p\right\rangle+\left\langle\gamma f(p)-A p, x_{t}-p\right\rangle\right) \\
& \leq \frac{\gamma \alpha}{\bar{\gamma}}\left\|x_{t}-p\right\|^{2}+\frac{1}{\bar{\gamma}}\left\langle\gamma f(p)-A p, x_{t}-p\right\rangle,
\end{aligned}
$$

which yields that

$$
\left\|x_{t}-p\right\|^{2} \leq \frac{1}{\bar{\gamma}-\alpha \gamma}\left\langle\gamma f(p)-A p, x_{t}-p\right\rangle .
$$

Since $H$ is a Hilbert space and $\left\{x_{t}\right\}$ is bounded as $t \rightarrow \infty$, there exists a sequence $\left\{t_{n}\right\}$ in $(0, \infty)$ such that $t_{n} \rightarrow \infty$ and $x_{t_{n}}-\bar{x} \in H$. By Lemma 3.1(b), we have $\left\|x_{t}-S(t) x_{t}\right\|$ $\rightarrow 0$ as $t \rightarrow \infty$. Since $\mathcal{T}$ has property $(\mathcal{A})$ with respect to the family $\mathcal{S}$, it follows that $x_{t}-T(\mathcal{T}) x_{t} \rightarrow 0$ as $t \rightarrow \infty$ for all $\tau>0$. Hence, by Lemma 2.2, $\bar{x} \in F(\mathcal{T}) \subseteq F(\mathcal{S})$. By (3.3), we see $x_{t_{n}} \rightarrow \bar{x}$. We next prove that $\bar{x}$ solves the variational inequality (3.2). From (3.1), we arrive at

$$
(A-\gamma f) x_{t}=-\frac{1}{t}(I-t A)\left[x_{t}-S(t) x_{t}\right] .
$$

For $p \in F(\mathcal{T})$, it follows from (2.4) that

$$
\begin{aligned}
& \left\langle(A-\gamma f) x_{t}, x_{t}-p\right\rangle=-\frac{1}{t}\left\langle(I-t A)\left[x_{t}-S(t) x_{t}\right], x_{t}-p\right\rangle \\
& =-\frac{1}{t}\left\langle\left[(I-S(t)) x_{t}-(I-S(t)) p\right], x_{t}-p\right\rangle+\left\langle A(I-S(t)) x_{t}, x_{t}-p\right\rangle \\
& \leq\left\langle A(I-S(t)) x_{t}, x_{t}-p\right\rangle .
\end{aligned}
$$


Since $x_{t_{n}} \rightarrow \bar{x}$, we obtain

$$
\langle(A-\gamma f) \bar{x}, \bar{x}-p\rangle \leq 0,
$$

i.e., $\bar{x}$ satisfies the variational inequality (3.2). By the uniqueness, it follows $\bar{x}=x^{*}$.

In a summary, we have shown that each cluster point of $\left\{x_{t}\right\}$ (as $t \rightarrow \infty$ ) equals $x^{*}$. Therefore, $x_{t} \rightarrow x^{*}$ as $t \rightarrow \infty$. The variational inequality (3.2) can be rewritten as

$$
\left\langle\left[(I-A+\gamma f) x^{*}\right]-x^{*}, x^{*}-p\right\rangle \geq 0, \quad p \in F(\mathcal{T}) .
$$

This, by Lemma 2.4, is equivalent to

$$
P_{F(\mathcal{T})}(I-A+\gamma f) x^{*}=x^{*} .
$$

This completes the proof. $\square$

Theorem 3.3. (Inexact explicit scheme) Let $H$ be a real Hilbert space $H$ and $\mathcal{T}=\{T(t): 0 \leq t<\infty\}$ be a family of nonexpansive self-mappings on $H$ such that $F(\mathcal{T}) \neq \emptyset, f: H \rightarrow H$ be an $\alpha$-contraction and $A$ be a strongly positive linear bounded self-adjoint operator on $H$ with the coefficient $\bar{\gamma}>0$. Let $\left\{t_{n}\right\}$ be a positive real divergent sequence and let $\Gamma=\left\{S_{t_{n}}: n \in \mathbb{N}\right\}$ be a sequence nonexpansive self-mappings on $H$ such that $F(\mathcal{T}) \subseteq \cap_{n \in \mathbb{N}} F\left(S_{t_{n}}\right)$. For $x_{0} \in H$, let $\left\{x_{n}\right\}$ be a sequence in $H$ generated by

$$
x_{n+1}=\alpha_{n} \gamma f\left(x_{n}\right)+\beta_{n} x_{n}+\left(\left(1-\beta_{n}\right) I-\alpha_{n} A\right) S_{t_{n}}\left(x_{n}\right)+e_{n}, n \geq 0
$$

where $\left\{\alpha_{n}\right\} \subset(0,1],\left\{\beta_{n}\right\} \subset[0,1]$, and $\left\{e_{n}\right\}$ is an error sequence in $H$ satisfying the following conditions:

(R1) $\lim _{n \rightarrow \infty} \alpha_{n}=\lim _{n \rightarrow \infty} \beta_{n}=0$ and $\sum_{n=0}^{\infty} \alpha_{n}=\infty$,

(R2) $\lim _{n \rightarrow \infty} \frac{\left\|e_{n}\right\|}{\alpha_{n}}=0$.

Assume that $0<\gamma<\bar{\gamma} / \alpha$ and that $\left\{S_{t_{n}}\left(x_{n}\right)\right\}$ is an approximating fixed point sequence of family $\mathcal{T}$. Assume that $x^{*} \in F(\mathcal{T})$, which solves the variational inequality (3.2). Then $\left\{x_{n}\right\}$ strongly converges to $x^{*}$.

Proof. Set $y_{n}=S_{t_{n}}\left(x_{n}\right)$. We divide the proof into three parts.

Step 1. Show the sequences $\left\{x_{n}\right\}$ and $\left\{y_{n}\right\}$ are bounded.

Noticing that $\lim _{n \rightarrow \infty} \alpha_{n}=\lim _{n \rightarrow \infty} \beta_{n}=0$, we may assume, with no loss of generality, that $\frac{\alpha_{n}}{1-\beta_{n}}<\|A\|^{-1}$ for all $n \geq 0$. From Lemma 2.7, we know that $\left\|\left(1-\beta_{n}\right) I-\alpha_{n} A\right\| \leq\left(1-\beta_{n}-\alpha_{n} \bar{\gamma}\right)$. Noticing that $x^{*} \in F(\mathcal{T})$, which solves the variational inequality (3.2). By assumption (R2), we have that $\left\{\frac{\left\|e_{n}\right\|}{\alpha_{n}}\right\}$ is bounded. Then, there exists a nonnegative real number $K$ such that

$$
\left\|\gamma f\left(x^{*}\right)-A x^{*}\right\|+\frac{\left\|e_{n}\right\|}{\alpha_{n}} \leq K \text { for all } n \geq 0 .
$$

From (3.4), we have

$$
\begin{aligned}
& \left\|x_{n+1}-x^{*}\right\| \\
& =\left\|\alpha_{n}\left(\gamma f\left(x_{n}\right)-A p\right)+\beta_{n}\left(x_{n}-x^{*}\right)+\left(\left(1-\beta_{n}\right) I-\alpha_{n} A\right)\left(S_{t_{n}}\left(x_{n}\right)-x^{*}\right)+e_{n}\right\| \\
& \leq \alpha_{n}\left\|\gamma f\left(x_{n}\right)-A x^{*}\right\|+\beta_{n}\left\|x_{n}-x^{*}\right\|+\left(1-\beta_{n}-\alpha_{n} \bar{\gamma}\right)\left\|S_{t_{n}}\left(x_{n}\right)-x^{*}\right\|+\left\|e_{n}\right\| \\
& \leq \alpha_{n} \gamma\left\|f\left(x_{n}\right)-f\left(x^{*}\right)\right\|+\alpha_{n}\|\gamma f(p)-A p\|+\beta_{n}\left\|x_{n}-x^{*}\right\| \\
& +\left(1-\beta_{n}-\alpha_{n} \bar{\gamma}\right)\left\|x_{n}-x^{*}\right\|+\left\|e_{n}\right\| \\
& \leq\left[1-\alpha_{n}(\bar{\gamma}-\gamma \alpha)\right]\left\|x_{n}-x^{*}\right\|+\alpha_{n}\left(\|\gamma f(p)-A p\|+\frac{\left\|e_{n}\right\|}{\alpha_{n}}\right) \\
& \leq\left[1-\alpha_{n}(\bar{\gamma}-\gamma \alpha)\right]\left\|x_{n}-x^{*}\right\|+\alpha_{n} K .
\end{aligned}
$$


By simple inductions, we see that

$$
\left\|x_{n}-x^{*}\right\| \leq \max \left\{\left\|x_{0}-x^{*}\right\|, \frac{K}{\bar{\gamma}-\gamma \alpha}\right\}=: R,
$$

which yields that the sequence $\left\{x_{n}\right\}$ is bounded. Note that

$$
\left\|y_{n}-x^{*}\right\| \leq\left\|x_{n}-x^{*}\right\|,
$$

and hence the sequence $\left\{y_{n}\right\}$ is bounded.

Step 2. Show that

$$
\limsup _{n \rightarrow \infty}\left\langle(\gamma f-A) x^{*}, y_{n}-x^{*}\right\rangle \leq 0,
$$

where $x^{*}$ is the solution of the variational inequality (3.2).

Let $D$ be the ball centered in $x^{*}$ and with radius $R$, i.e.,

$$
D:=\left\{w \in H:\left\|w-x^{*}\right\| \leq \max \left\{\left\|x_{0}-x^{*}\right\|, \frac{K}{\bar{\gamma}-\gamma \alpha}\right\}\right\} .
$$

From (3.5) we see that $D$ is a nonempty closed convex bounded subset of $H$ which is $T(t)$-invariant for each $t \in[0, \infty)$ and contain $\left\{x_{n}\right\}$. Therefore, we assume, without loss of generality, $\mathcal{T}=\{T(t): 0 \leq t<\infty\}$ is a family nonexpansive self-mappings on $D$.

Taking a suitable subsequence $\left\{y_{n_{i}}\right\}$ of $\left\{y_{n}\right\}$, we see that

$$
\limsup _{n \rightarrow \infty}\left\langle(\gamma f-A) x^{*}, y_{n}-x^{*}\right\rangle=\lim _{i \rightarrow \infty}\left\langle(\gamma f-A) x^{*}, y_{n_{i}}-x^{*}\right\rangle .
$$

Since the sequence $\left\{y_{n}\right\}$ is also bounded, we may assume that $y_{n_{i}} \rightarrow \bar{x}$. Note that $\left\{y_{n}\right\}$ is an approximating fixed point sequence of family $\mathcal{T}$, i.e.,

$$
\lim _{n \rightarrow \infty}\left\|y_{n}-T(h) y_{n}\right\|=0 \text { for all } 0 \leq h<\infty
$$

Using (3.7) we obtain, from the demiclosedness principle, that $\bar{x} \in F(\mathcal{T})$. Therefore, we have

$$
\limsup _{n \rightarrow \infty}\left\langle(\gamma f-A) x^{*}, y_{n}-x^{*}\right\rangle=\left\langle(\gamma f-A) x^{*}, \bar{x}-x^{*}\right\rangle \leq 0 .
$$

On the other hand, we have

$$
\left\|x_{n+1}-y_{n}\right\| \leq \alpha_{n}\left\|\gamma f\left(x_{n}\right)-A x_{n}\right\|+\beta_{n}\left\|x_{n}-y_{n}\right\| .
$$

From the assumption $\lim _{n \rightarrow \infty} \alpha_{n}=\lim _{n \rightarrow \infty} \beta_{n}=0$ that $\lim _{n \rightarrow \infty}\left\|x_{n+1}-y_{n}\right\|=0$, which combines with (3.8) gives that

$$
\limsup _{n \rightarrow \infty}\left\langle(\gamma f-A) x^{*}, x_{n+1}-x^{*}\right\rangle \leq 0 .
$$

Step 3. Show $x_{n} \rightarrow x^{*}$ as $n \rightarrow \infty$.

Since the sequence $\left\{x_{n}\right\}$ is bounded, we may assume a nonnegative real number $L$ such that $\left\|x_{n}-x^{*}\right\| \leq L$ for all $n \geq 0$. Note that 


$$
\begin{aligned}
&\left\|x_{n+1}-x^{*}\right\|^{2} \\
&=\left\langle\alpha_{n}\left(\gamma f\left(x_{n}\right)-A x^{*}\right)+\beta_{n}\left(x_{n}-x^{*}\right)+\left(\left(1-\beta_{n}\right) I-\alpha_{n} A\right)\left(y_{n}-x^{*}\right)+e_{n}, x_{n+1}-x^{*}\right\rangle \\
&= \alpha_{n}\left\langle\gamma f\left(x_{n}\right)-A x^{*}, x_{n+1}-x^{*}\right\rangle+\beta_{n}\left\langle x_{n}-x^{*}, x_{n+1}-x^{*}\right\rangle \\
&+\left\langle\left(\left(1-\beta_{n}\right) I-\alpha_{n} A\right)\left(y_{n}-x^{*}\right)+e_{n}, x_{n+1}-x^{*}\right\rangle \\
& \leq \alpha_{n}\left(\gamma\left\langle f\left(x_{n}\right)-f\left(x^{*}\right), x_{n+1}-x^{*}\right\rangle+\left\langle\gamma f\left(x^{*}\right)-A x^{*}, x_{n+1}-x^{*}\right\rangle\right) \\
&+\beta_{n}\left\|x_{n}-x^{*}\right\|\left\|x_{n+1}-x^{*}\right\|+\left\|\left(1-\beta_{n}\right) I-\alpha_{n} A\right\|\left\|y_{n}-x^{*}\right\|\left\|x_{n+1}-x^{*}\right\|+\left\|e_{n}\right\| L \\
& \leq \alpha_{n} \alpha \gamma\left\|x_{n}-x^{*}\right\|\left\|x_{n+1}-x^{*}\right\|+\alpha_{n}\left\langle\gamma f\left(x^{*}\right)-A x^{*}, x_{n+1}-x^{*}\right\rangle \\
&+\beta_{n}\left\|x_{n}-x^{*}\right\|\left\|x_{n+1}-x^{*}\right\|+\left(1-\beta_{n}-\alpha_{n} \bar{\gamma}\right)\left\|x_{n}-x^{*}\right\|\left\|x_{n+1}-x^{*}\right\|+\left\|e_{n}\right\| L \\
&= {\left[1-\alpha_{n}(\bar{\gamma}-\gamma \alpha)\right]\left\|x_{n}-x^{*}\right\|\left\|x_{n+1}-x^{*}\right\|+\alpha_{n}\left\langle\gamma f\left(x^{*}\right)-A x^{*}, x_{n+1}-x^{*}\right\rangle+\left\|e_{n}\right\| L } \\
& \leq \frac{1-\alpha_{n}(\bar{\gamma}-\gamma \alpha)}{2}\left(\left\|x_{n}-x^{*}\right\|^{2}+\left\|x_{n+1}-x^{*}\right\|^{2}\right)+\alpha_{n}\left\langle\gamma f\left(x^{*}\right)-A x^{*}, x_{n+1}-x^{*}\right\rangle+\left\|e_{n}\right\| L \\
& \leq \frac{1-\alpha_{n}(\bar{\gamma}-\gamma \alpha)}{2}\left\|x_{n}-x^{*}\right\|^{2}+\frac{1}{2}\left\|x_{n+1}-x^{*}\right\|^{2}+\alpha_{n}\left\langle\gamma f\left(x^{*}\right)-A x^{*}, x_{n+1}-x^{*}\right\rangle+\left\|e_{n}\right\| L .
\end{aligned}
$$

It follows that

$$
\left\|x_{n+1}-x^{*}\right\|^{2} \leq\left[1-\alpha_{n}(\bar{\gamma}-\gamma \alpha)\right]\left\|x_{n}-x^{*}\right\|^{2}+\alpha_{n}\left(2\left\langle\gamma f\left(x^{*}\right)-A x^{*}, x_{n+1}-x^{*}\right\rangle+\frac{2\left\|e_{n}\right\|}{\alpha_{n}} L\right) .
$$

By using Lemma 2.8, we can obtain the desired conclusion easily. $\square$

\section{Applications}

\subsection{Applications to zeros of maximal monotone operators}

Let $\mathrm{H}$ be a real Hilbert space. Let $\mathbb{A} \subset H \times H$ be an operator on $H$. The set $D(\mathbb{A})$ defined by $D(\mathbb{A})=\{x \in H: \mathbb{A} x \neq \emptyset\}$ is called the domain of $\mathbb{A}$, the set $R(\mathbb{A})$ defined by $R(\mathbb{A})=\cup_{x \in X} \mathbb{A} x$ is called the range of $\mathbb{A}$ and the set $G(\mathbb{A})$ defined by $G(\mathbb{A})=\{(x, y) \in H \times H: x \in D(\mathbb{A}), y \in \mathbb{A} x\}$ is called the graph of $\mathbb{A}$. An operator $\mathbb{A} \subset H \times H$ with domain $D(\mathbb{A})$ is said to be monotone if for each $x_{i} \in D(\mathbb{A})$ and $y_{i} \in \mathbb{A} x_{i}(i=1,2)$, we have $\left\langle x_{1}-x_{2}, y_{1}-y_{2}\right\rangle \geq 0$. A monotone operator $\mathbb{A}$ is said to be maximal monotone if the graph $G(\mathbb{A})$ is not properly contained in the graph of any other monotone operator on $H$. If $\mathbb{A}: H \rightarrow 2^{H}$ is maximal monotone, then we can define, for each $\lambda>0$, a nonexpansive single-valued mapping $J_{\lambda}^{\mathbb{A}}: H \rightarrow H$ by $J_{\lambda}^{\mathbb{A}}=(I+\lambda \mathbb{A})^{-1}$. It is called the resolvent of $\mathbb{A}$. Let $\mathcal{N}(\mathbb{A})=\mathbb{A}^{-1} 0=\{x \in D(\mathbb{A}): 0 \in \mathbb{A} x\}$. It is known that $\mathcal{N}(\mathbb{A})$ is closed and convex.

Lemma 4.1. ([22]) Let $\mathbb{A} \subset H \times H$ be a maximal monotone operator. Then

$$
\frac{1}{r}|| J_{t} x-J_{r}^{\mathbb{A}} J_{t}^{\mathbb{A}} x\left\|\leq \frac{1}{t}\right\| x-J_{t}^{\mathbb{A}} x \| \text { for all } x \in H \text { and } r, t>0 .
$$

Proposition 4.2 shows that the family $\left\{J_{t}^{\mathbb{A}}: t>0\right\}$ of resolvent operators of a maximal monotone operator $\mathbb{A}$ enjoys property $(\mathcal{A})$.

Proposition 4.2. Let $\mathbb{A} \subset H \times H b e$ a maximal monotone operator. Let $\left\{z_{t}\right\}_{t>0} \in \mathcal{B}(H)$ such that $\left\|z_{t}-J_{t}^{\mathbb{A}} z_{t}\right\| \rightarrow 0$ as $t \rightarrow \infty$. Then $\left\|z_{t}-J_{r}^{\mathbb{A}} z_{t}\right\| \rightarrow 0$ as $t \rightarrow \infty$ for each $r>0$.

Proof. Let $r, t>0$. By Lemma 4.1, we have

$$
\frac{1}{r}|| J_{t}^{\mathbb{A}} z_{t}-J_{r}^{\mathbb{A}} J_{t}^{\mathbb{A}} z_{t}\left\|\leq \frac{1}{t}\right\| z_{t}-J_{t}^{\mathbb{A}} z_{t} \| .
$$


Using (4.1), we have

$$
\begin{aligned}
\left\|z_{t}-J_{r}^{\mathbb{A}} z_{t}\right\| & \leq\left\|z_{t}-J_{t}^{\mathbb{A}} z_{t}\right\|+\left\|J_{t}^{\mathbb{A}} z_{t}-J_{r}^{\mathbb{A}} J_{t}^{\mathbb{A}} z_{t}\right\|+\left\|J_{r}^{\mathbb{A}} J_{t}^{\mathbb{A}} z_{t}-J_{r}^{\mathbb{A}} z_{t}\right\| \\
& \leq\left(2+\frac{r}{t}\right)\left\|z_{t}-J_{t}^{\mathbb{A}} z_{t}\right\| \rightarrow 0 \text { as } t \rightarrow \infty
\end{aligned}
$$

Given a monotone operator $\mathbb{A} \subset H \times H$, we consider the following problem of finding $z \in H$ such that:

$0 \in \mathbb{A} z$. $(\mathrm{P})$

The Problem $(P)$ can be regarded as a unified formulation of several important problems. For an appropriate choice of the operator A, Problem $(P)$ covers a wide range of mathematical applications; for example, variational inequalities, complementarity problems, and non-smooth convex optimization. Problem $(P)$ has applications in physics, economics, and in several areas of engineering. Therefore, one of the most interesting and important problems in the theory of maximal monotone operators is to find an efficient iterative algorithm to compute approximately zeroes of maximal monotone operators. One method for finding zeros of maximal monotone operators is the proximal point algorithm. Let A be a maximal monotone operator in a Hilbert space $H$. The proximal point algorithm generates, for starting $x_{1} \in H$, a sequence $\left\{x_{n}\right\}$ in $H$ by

$$
x_{n+1}=J_{r_{n}}^{\mathbb{A}} x_{n} \text { for all } n \in \mathbb{N},
$$

where $\left\{r_{n}\right\}$ is a regularization sequence in $(0, \infty)$. Note that $(4.2)$ is equivalent to

$$
0 \in \frac{1}{r_{n}}\left(x_{n+1}-x_{n}\right)+\mathbb{A} x_{n+1} \text { for all } n \in \mathbb{N} .
$$

This was first introduced by Martinet [23]. If $\psi: H \rightarrow \mathbb{R} \cup\{\infty\}$ is a proper lower semicontinuous convex function, then the algorithm reduces to

$$
x_{n+1}=\underset{y \in H}{\arg \min }\left\{\psi(y)+\frac{1}{2 r_{n}}\left\|x_{n}-y\right\|^{2}\right\} \text { for all } n \in \mathbb{N} .
$$

Rockafellar [24] studied the proximal point algorithm in the framework of Hilbert space and he proved the following:

Theorem 4.3. Let $H$ be a Hilbert space and $\mathbb{A} \subset H \times H a$ maximal monotone operator. Let $\left\{x_{n}\right\}$ be a sequence in $H$ defined by (4.2), where $\left\{r_{n}\right\}$ is a sequence in $(0, \infty)$ such that $\lim \inf _{n \rightarrow \infty} r_{n}>0$. If $\mathbb{A}^{-1} 0 \neq \emptyset$, then the sequence $\left\{x_{n}\right\}$ converges weakly to an element of $\mathbb{A}^{-1} 0$.

Rockafellar [24] has given a more practical method which is an inexact variant of the method

$$
e_{n} \in x_{n}-x_{n-1}+r_{n} A x_{n}
$$

where $\left\{e_{n}\right\}$ is regarded as an error sequence. The method is called inexact proximal point algorithm. It was shown in Rockafellar [24] that if $e_{n} \rightarrow 0$ quickly enough such that $\sum_{n=1}^{\infty}\left\|e_{n}\right\|<\infty$, then $x_{n}-z \in H$ with $0 \in A(z)$. In 2002, Xu [12] modified the proximal point algorithm for solving Problem $(P)$ and gave strong convergence of the algorithm in a Hilbert space setting under the same assumption $\sum_{n=1}^{\infty}\left\|e_{n}\right\|<\infty$. 
The criteria $\sum_{n=1}^{\infty}\left\|e_{n}\right\|<\infty$ imposed for convergence of inexact proximal point algorithms (see $[12,24])$ is somewhat undesirable, because it impose increasing precision along the iterative process. This brings us to the following natural question:

Question 4.4. Is it possible to further modify inexact proximal point algorithm without the assumption $\sum_{n=1}^{\infty}\left\|e_{n}\right\|<\infty$, so that it can generate a strongly convergent sequence?

Recently, Sahu and Yao [25] introduced and studied the prox-Tikhonov method for solving Problem $(\mathrm{P})$ in the Banach space setting and they partially answered Question 4.4. We now establish more general results in the Hilbert space setting:

Theorem 4.5. Let $H$ be a real Hilbert space $H$. Let $\mathbb{A} \subset H \times H$ be a maximal monotone operator with $\mathcal{N}(\mathbb{A}) \neq \emptyset, f: H \rightarrow H$ an $\alpha$-contraction and $A$ a strongly positive linear bounded self-adjoint operator on $H$ with the coefficient $\bar{\gamma}>0$. Assume that $0<\gamma<\bar{\gamma} / \alpha$ and that $\left\{b_{t}: t>0\right\}$ is a net in $\left(0,\|A\|^{-1}\right)$ such that $\lim _{t \rightarrow \infty} b_{t}=0$. Then $\left\{x_{t}\right\}$ defined by

$$
x_{t}=b_{t} \gamma f\left(x_{t}\right)+\left(I-b_{t} A\right) J_{t}^{\mathbb{A}} x_{t} .
$$

strongly converges as $t \rightarrow \infty$ to $x^{*} \in \mathcal{N}(\mathbb{A})$, where $x^{*} \in P_{\mathcal{N}(\mathbb{A})}(I-A+\gamma f) x^{*}$ is a solution of the following variational inequality:

$$
\left\langle(\gamma f-A) x^{*}, p-x^{*}\right\rangle \leq 0, \forall p \in \mathcal{N}(\mathbb{A}) .
$$

Proof. Set $T(t):=J_{t}^{\mathbb{A}}$ for $t>0$. Then $\{T(t): t>0\}$ is a family of nonexpansive mappings with $F(T(t))=\mathcal{N}(\mathbb{A})$ for each $t>0$. Proposition 4.2 shows that the family $\left\{J_{t}^{\mathbb{A}}: t>0\right\}$ of resolvent operators enjoys property $(\mathcal{A})$. Therefore, Theorem 4.5 follows from Theorem 3.2. $\square$

Theorem 4.6. Let $H$ be a real Hilbert space $H$. Let $\mathbb{A} \subset H \times H b e$ a maximal monotone operator with $\mathcal{N}(\mathbb{A}) \neq \emptyset, f: H \rightarrow H$ an $\alpha$-contraction and $A$ a strongly positive linear bounded self-adjoint operator on $H$ with the coefficient $\bar{\gamma}>0$. Assume that $0<\gamma<\bar{\gamma} / \alpha$ and $\left\{t_{n}\right\}$ is a positive real divergent sequence. For $x_{0} \in H$, let $\left\{x_{n}\right\}$ be a sequence in $H$ generated by

$$
x_{n+1}=\alpha_{n} \gamma f\left(x_{n}\right)+\beta_{n} x_{n}+\left(\left(1-\beta_{n}\right) I-\alpha_{n} A\right) J_{t_{n}}^{\mathbb{A}}\left(x_{n}\right)+e_{n}, \quad n \geq 0
$$

where $\left\{\alpha_{n}\right\} \subset(0,1],\left\{\beta_{n}\right\} \subset[0,1]$ and $\left\{e_{n}\right\}$ is an error sequence in $H$ satisfying conditions $(R 1)$ and $(R 2)$. Then $\left\{x_{n}\right\}$ strongly converges to $x^{*}$, where $x^{*}=P_{\mathcal{N}(\mathbb{A})}(I-A+\gamma f) x^{*}$ is a solution of the variational inequality (4.3).

Proof. Set $S_{t_{n}}:=J_{t_{n}}^{\mathbb{A}}$ and $y_{n}:=S_{s_{n}}\left(x_{n}\right)$. Then it remains to show that $\left\{y_{n}\right\}$ is an approximating fixed point sequence of the family $\left\{J_{t}^{\mathbb{A}}: t>0\right\}$ of resolvent operators of $\mathbb{A}$. As in the proof of Theorem 3.3, one can show that $\left\{x_{n}\right\}$ and $\left\{y_{n}\right\}$ are bounded. Then, there positive real number $M$ such that $\left\|x_{n}-J_{t_{n}}^{\mathbb{A}} x_{n}\right\| \leq M$ for all $n \geq 0$. For any fixed $r>0$, by Lemma 4.1, we have

$$
\begin{aligned}
\left\|J_{t_{n}}^{\mathbb{A}} x_{n}-J_{r}^{\mathbb{A}} J_{t_{n}}^{\mathbb{A}} x_{n}\right\| & \leq \frac{r}{t_{n}}\left\|x_{n}-J_{t_{n}}^{\mathbb{A}} x_{n}\right\| \\
& \leq \frac{r}{t_{n}} M .
\end{aligned}
$$


Thus, in particular, we derive

$$
\left\|y_{n}-J_{r}^{\mathbb{A}} y_{n}\right\| \rightarrow 0 \text { as } n \rightarrow \infty
$$

for all $r>0$. Therefore, Theorem 4.6 follows from Theorem 3.3.

Theorem 4.6 is more general than results of Kamimura and Takahashi [26] and Xu [12]. In particular, Theorem 4.6 provides an affirmative answer of Question 4.4 in the context of finding solution of variational inequality (4.3).

\subsection{Applications to equilibrium problems}

Let $H$ be a Hilbert space and $G: H \times H \rightarrow \mathbb{R}$ be an equilibrium function, that is

$$
G(u, u)=0 \quad \text { for every } u \in H .
$$

The equilibrium problem is defined as follows,

$$
\text { Find } \tilde{x} \in H \text { such that } G(x, y) \geq 0 \text { for all } y \in H \text {. }
$$

A solution $\tilde{x}$ of the equilibrium problem is called an equilibrium point and the set of all equilibrium points will be denoted by $\operatorname{EP}(G)$. The topic has been considered by several authors (see $[27,28]$ ). We shall assume some mild conditions over $G$ in such a way that results can be applied in several cases including optimization problems, fixed point problems, variational problems, variational inequality problems, and convex vector minimization problems $[29,30]$.

Lemma 4.7. ([29]) Let $C$ be a nonempty closed convex subset of $H$ and $G: C \times C \rightarrow \mathbb{R}$ satisfy,

(A1) for all $x \in C, G(x, x)=0$;

(A2) $G$ is monotone, i.e., $G(x, y)+G(y, x) \leq 0$ for all $x, y \in C$;

(A3) for all $x, y, z \in C$,

$$
\lim \sup G(t z+(1-t) x, y) \leq G(x, y) \text { as } t \rightarrow 0 ;
$$

(A4) for all $x \in C, y \alpha G(x, y)$ is convex and lower semicontinuous.

For $\times \in C$ and $r>0$, set $S_{r}: H \rightarrow C$ to be the resolvent for $G$,

$$
S_{r}(x):=\left\{z \in C: G(z, y)+\frac{1}{r}\langle y-z, z-x\rangle \geq 0, \forall y \in C\right\},
$$

then $S_{r}$ is well defined and the following hold:

(1) $S_{r}$ is single-valued;

(2) $S_{r}$ is firmly nonexpansive, i.e.,

$$
\left\|S_{r} x-S_{r} y\right\|^{2} \leq\left\langle S_{r} x-S_{r} y, x-y\right\rangle,
$$

for all $x, y \in H$;

(3) $F\left(S_{r}\right)=E P(G)$;

(4) $E P(G)$ is closed and convex.

In order to show that the family $\left\{S_{t}: t>0\right\}$ of resolvent operators of $G$ enjoys property $(\mathcal{A})$, we need the following technical lemma.

Lemma 4.8. Let $G$ be an equilibrium function satisfying the assumptions of Lemma 4.7. Then 


$$
\left\|S_{t}(x)-S_{r} S_{t}(x)\right\| \leq \frac{r}{t}\left\|x-S_{t}(x)\right\|
$$

for all $\times \in H$ and $r, t>0$.

Proof. Let $x, y \in H$ and $r, t>0$. By the definition of $S_{t}$, we have

$$
G\left(S_{t}(x), z\right)+\frac{1}{t}\left\langle z-S_{t}(x), S_{t}(x)-x\right\rangle \geq 0, \forall z \in H
$$

and

$$
G\left(S_{r}(y), z\right)+\frac{1}{r}\left\langle z-S_{r}(\gamma), S_{r}(\gamma)-\gamma\right\rangle \geq 0, \forall z \in H .
$$

Put $z=S_{r}(y)$ in (4.4) and $z=S_{t}(x)$ in (4.5), we obtain

$$
G\left(S_{t}(x), S_{r}(y)\right)+\frac{1}{t}\left\langle S_{r}(y)-S_{t}(x), S_{t}(x)-x\right\rangle \geq 0
$$

and

$$
G\left(S_{r}(\gamma), S_{t}(x)\right)+\frac{1}{r}\left\langle S_{t}(x)-S_{r}(y), S_{r}(\gamma)-\gamma\right\rangle \geq 0,
$$

respectively. Since $G$ is monotone, from (4.6) and (4.7), we have

$$
\left\langle S_{t}(x)-S_{r}(y), \frac{S_{r}(y)-y}{r}-\frac{S_{t}(x)-x}{t}\right\rangle \geq 0
$$

Set $y=S_{t}(x)$ in (4.8), we get

$$
\left\langle S_{t}(x)-S_{r} S_{t}(x), \frac{S_{r} S_{t}(x)-S_{t}(x)}{r}-\frac{S_{t}(x)-x}{t}\right\rangle \geq 0
$$

and hence

$$
\frac{1}{r}\left\|S_{t}(x)-S_{r} S_{t}(x)\right\|^{2} \leq\left\langle S_{t}(x)-S_{r} S_{t}(x), \frac{x-S_{t}(x)}{t}\right\rangle \leq \frac{1}{t}\left\|S_{t}(x)-S_{r} S_{t}(x)\right\|\left\|x-S_{t}(x)\right\| .
$$

Therefore,

$$
\left\|S_{t}(x)-S_{r} S_{t}(x)\right\| \leq \frac{r}{t}\left\|x-S_{t}(x)\right\| .
$$

口

From this, we deduce the property $(\mathcal{A})$ for the family $\left\{S_{t}: t>0\right\}$ of resolvent operators of $G$.

Lemma 4.9. Let $G$ be an equilibrium function satisfying the assumptions of Lemma 4.7. Then the family $\left\{S_{t}: t>0\right\}$ enjoys property $(\mathcal{A})$.

Proof. Let $\left\{z_{t}\right\} \in \mathcal{B}(H)$ such that $z_{t}-S_{t} z_{t} \rightarrow 0$. Then, for any fixed $r>0$,

$$
\begin{aligned}
\left\|z_{t}-S_{r} z_{t}\right\| & \leq\left\|z_{t}-S_{t} z_{t}\right\|+\left\|S_{t} z_{t}-S_{r} S_{t} z_{t}\right\|+\left\|S_{r} S_{t} z_{t}-S_{r} z_{t}\right\| \\
& \leq\left(2+\frac{r}{t}\right)\left\|z_{t}-S_{t} z_{t}\right\|
\end{aligned}
$$

by nonexpansivity and Lemma 4.8. In particular, we derive that $z_{t}-S_{r} z_{t} \rightarrow 0$ as $t \rightarrow$ $\infty$. $\square$

From this last and from Theorem 3.2, we have 
Theorem 4.10. Let $H$ be a real Hilbert space $H$. Let $G: H \times H \rightarrow \mathbb{R} b$ e an equilibrium function satisfying the assumptions of Lemma 4.7 and let $\left\{S_{t}: t>0\right\}$ be the family of resolvent operators for $G$. Let $f: H \rightarrow H$ be an $\alpha$-contraction and $A$ be a strongly positive linear bounded self-adjoint operator on $H$ with the coefficient $\bar{\gamma}>0$. Assume that $\operatorname{EP}(G) \neq \varnothing$ and $0<\gamma<\bar{\gamma} / \alpha$. Let $\left\{b_{t}: t>0\right\}$ be a net in $\left(0,\|A\|^{-1}\right)$ such that $\lim _{t \rightarrow \infty} b_{t}=0$. Then $\left\{x_{t}\right\}$ defined by

$$
x_{t}=b_{t} \gamma f\left(x_{t}\right)+\left(I-b_{t} A\right) S_{t} x_{t} .
$$

strongly converges as $t \rightarrow \infty$ to $x^{*} \in E P(G)$, where $x^{*}=P_{E P(G))}(I-A+\gamma f) x^{*}$ is a solution of the following variational inequality:

$$
\left\langle(\gamma f-A) x^{*}, p-x^{*}\right\rangle \leq 0, \quad \forall p \in E P(G) .
$$

Proof. Note that $\left\{S_{t}: t>0\right\}$ is a family of resolvent operators for $G$ such that $F\left(S_{t}\right)=$ $E P(G)$ for each $t>0$. Lemma 4.9 shows that the family $\left\{S_{t}: t>0\right\}$ of resolvent operators of $G$ enjoys property $(\mathcal{A})$. Therefore, Theorem 4.10 follows from Theorem 3.2. $\square$

Theorem 4.11. Let $H$ be a real Hilbert space $H$. Let $G: H \times H \rightarrow \mathbb{R} b$ e an equilibrium function satisfying the assumptions of Lemma 4.7 and let $\left\{S_{t}: t>0\right\}$ be the family of resolvent operators for $G$ such that $E P(G) \neq \varnothing$. Let $f: H \rightarrow H$ be an $\alpha$-contraction and $A$ be a strongly positive linear bounded self-adjoint operator on $H$ with the coefficient $\bar{\gamma}>0$. Assume that $0<\gamma<\bar{\gamma} / \alpha$ and $\left\{t_{n}\right\}$ is a positive real divergent sequence. For $x_{0} \in H$, let $\left\{x_{n}\right\}$ be a sequence in $H$ generated by

$$
x_{n+1}=\alpha_{n} \gamma f\left(x_{n}\right)+\beta_{n} x_{n}+\left(\left(1-\beta_{n}\right) I-\alpha_{n} A\right) S_{t_{n}}\left(x_{n}\right)+e_{n}, \quad n \geq 0
$$

where $\left\{\alpha_{n}\right\} \subset(0,1],\left\{\beta_{n}\right\} \subset[0,1]$ and $\left\{e_{n}\right\}$ is an error sequence in $H$ satisfying conditions $(R 1)$ and $(R 2)$. Then $\left\{x_{n}\right\}$ strongly converges to $x^{*} \in E P(G)$, where $x^{*}=P_{E P(G))}(I-$ $A+\gamma f) x^{*}$ is a solution of the variational inequality (4.10).

Proof. Set $y_{n}:=S_{t_{n}}\left(x_{n}\right)$. Then it remains to show that $\left\{y_{n}\right\}$ is an approximating fixed point sequence of the family $\left\{S_{t}: t>0\right\}$ of resolvent operators of $G$. As in the proof of Theorem 3.3, one can show that $\left\{z_{n}\right\}$ and $\left\{S_{t_{n}}\left(x_{n}\right)\right\}$ are bounded. Then, there positive real number $M$ such that $\left\|x_{n}-S_{t_{n}} x_{n}\right\| \leq M$ for all $n \geq 0$. For any fixed $r>0$, by Lemma 4.8, we have

$$
\left\|S_{t_{n}} x_{n}-S_{r} S_{t_{n}} x_{n}\right\| \leq \frac{r}{t_{n}} M .
$$

In particular, we derive $\left\|y_{n}-S_{r} y_{n}\right\| \rightarrow 0$ as $n \rightarrow \infty$. for all $r>0$. Therefore, Theorem 4.11 follows from Theorem 3.3. $\square$

Theorem 4.11 extends the corresponding result of Song et al. [31] in the context of the variational inequality (4.10).

\subsection{Applications to common fixed point problems}

In this section, we deduce some results by using Theorems 3.2 and 3.3. As a direct consequence of Theorem 4.12, we first have the following result.

Theorem 4.12. Let $H$ be a real Hilbert space $H$ and $\mathcal{T}=\{T(t): 0 \leq t<\infty\}$ be a nonexpansive semigroup on $H$ such that $F(\mathcal{T}) \neq \emptyset$. Let $f: H \rightarrow H$ be an $\alpha$-contraction and $A$ be a strongly positive linear bounded self-adjoint operator on $H$ with the coefficient $\bar{\gamma}>0$. Assume that $0<\gamma<\bar{\gamma} / \alpha$ and that $\left\{b_{t}: t>0\right\}$ is a net in $\left(0,\|A\|^{-1}\right)$ such that $\lim _{t \rightarrow \infty} b_{t}=0$. Then $\left\{x_{t}\right\}$ define by 


$$
x_{t}=b_{t} \gamma f\left(x_{t}\right)+\left(I-b_{t} A\right) \frac{1}{t} \int_{0}^{t} T(s) x_{t} d s
$$

strongly converges as $t \rightarrow \infty$ to $x^{*} \in F(\mathcal{T})$, where $x^{*}=P_{F(\mathcal{T})}(I-A+\gamma f) x^{*}$ is a solution of the variational inequality (3.2).

Proof. Example 2.12 implies that nonexpansive semigroup $\mathcal{T}=\left\{T(t): t \in \mathbb{R}^{+}\right\}$has property $(\mathcal{A})$ with respect to a nonexpansive semigroup $\left\{\sigma_{t}: t \in \mathbb{R}^{+}\right\}$. Therefore, Theorem 4.12 follows from Theorem 3.2.

Remark 4.13. Theorem 4.12 which include the corresponding results of Shioji and Takahashi [16] as a special case is reduced to Plubtieng and Punpaeng [14]when A = I, the identity mapping and $\gamma=1$.

Theorem 4.14. Let $H$ be a real Hilbert space $H$ and $\mathcal{T}=\{T(t): 0 \leq t<\infty\} a$ nonexpansive semigroup such that $F(\mathcal{T}) \neq \emptyset$. Let $f: H \rightarrow H$ be an $\alpha$-contraction and $A$ a strongly positive linear bounded self-adjoint operator on $H$ with the coefficient $\bar{\gamma}>0$. Assume that $0<\gamma<\bar{\gamma} / \alpha$ and $\left\{t_{n}\right\}$ is a positive real divergent sequence. For $x_{0} \in H$, let $\left\{x_{n}\right\}$ be a sequence in $H$ generated by

$$
x_{n+1}=\alpha_{n} \gamma f\left(x_{n}\right)+\beta_{n} x_{n}+\left(\left(1-\beta_{n}\right) I-\alpha_{n} A\right) \frac{1}{t_{n}} \int_{0}^{t_{n}} T(s) x_{n} d s+e_{n}, \quad n \geq 0
$$

where $\left\{\alpha_{n}\right\} \subset(0,1],\left\{\beta_{n}\right\} \subset[0,1]$ and $\left\{e_{n}\right\}$ is an error sequence in $H$ satisfying conditions $(R 1)$ and $(R 2)$. Then $\left\{x_{n}\right\}$ strongly converges to $x^{*} \in F(\mathcal{T})$, where $x^{*}=P_{F(\mathcal{T})}(I-A+\gamma f) x^{*}$ is a solution of the variational inequality (3.2).

Proof. For each $n \in \mathbb{N}$, define $y_{n}=S_{t_{n}}\left(x_{n}\right)$. Note that $\left\{y_{n}\right\}$ is in a bounded set $D$ defined by (3.6). As in the the proof of Theorem 3.3, $\mathcal{T}=\{T(t): 0 \leq t<\infty\}$ is a semigroup of nonexpansive self-mappings on $D$. It follows from Lemma 2.1 that $\left\{y_{n}\right\}$ is an approximating fixed point sequence of semigroup $\mathcal{T}$.

Remark 4.15. If $\gamma=1$ and $A=I$, the identity mapping, then Corollary 2.4 is reduced to Theorem 3.3 of Plubtieng and Punpaeng [14].

If the sequence $\left\{\beta_{n}\right\} \equiv 0$, then Theorem 4.14 reduces to the following:

Corollary 4.16. Let $H$ be a real Hilbert space $H$ and $\mathcal{T}=\{T(t): 0 \leq t<\infty\}$ be a nonexpansive semigroup such that $F(\mathcal{T}) \neq \emptyset$. Let $f: H \rightarrow H$ be an $\alpha$-contraction and $A$ be a strongly positive linear bounded self-adjoint operator on $H$ with the coefficient $\bar{\gamma}>0$. Assume that $0<\gamma<\bar{\gamma} / \alpha$ and $\left\{t_{n}\right\}$ is a positive real divergent sequence. For $x_{0} \in H$, let $\left\{x_{n}\right\}$ be a sequence in $H$ generated by

$$
x_{n+1}=\alpha_{n} \gamma f\left(x_{n}\right)+\left(I-\alpha_{n} A\right) \frac{1}{t_{n}} \int_{0}^{t_{n}} T(s) x_{n} d s+e_{n}, n \geq 0
$$

where $\left\{\alpha_{n}\right\} \subset(0,1]$ and $\left\{e_{n}\right\}$ is an error sequence in $H$ satisfying the following conditions:

(R3) $\lim _{n \rightarrow \infty} \alpha_{n}=0$ and $\sum_{n=0}^{\infty} \alpha_{n}=\infty$,

(R4). $\lim _{n \rightarrow \infty} \frac{\left\|e_{n}\right\|}{\alpha_{n}}=0$

Then $\left\{x_{n}\right\}$ strongly converges to $x^{*} \in F(\mathcal{T})$, where $x^{*}=P_{F(\mathcal{T})}(I-A+\gamma f) x^{*}$ is a solution of the variational inequality (3.2). 
Remark 4.17. Corollary 4.16 includes Theorem 2 of Shimizu and Takahashi [17]as a special case.

Remark 4.18. Theorem 2.2 and Corollary 4.16 improve Theorems 3.2 and 3.4 of Marino and Xu [10]from a single nonexpansive mapping to a nonexpansive semigroup, respectively.

Using [[17], Lemma 1], we derive the following result, which generalizes Theorem 1 of Shimizu and Takahashi [17].

Corollary 4.19. Let $H$ be a real Hilbert space $H$ and let $S, T: H \rightarrow H$ be two commuting nonexpansive mappings such that $F(S) \cap F(T) \neq \varnothing$. Let $f: H \rightarrow H$ be an $\alpha$-contraction and $A$ be a strongly positive linear bounded self-adjoint operator on $H$ with the coefficient $\bar{\gamma}>0$ Assume that $0<\gamma<\bar{\gamma} / \alpha$. For $x_{0} \in H$, let $\left\{x_{n}\right\}$ be a sequence in $H$ generated by

$$
x_{n+1}=\alpha_{n} \gamma f\left(x_{n}\right)+\left(I-\alpha_{n} A\right) \frac{2}{(n+1)(n+2)} \sum_{k=0}^{n} \sum_{i+j=k} S^{i} T^{j} x_{n}+e_{n}, \quad n \geq 0
$$

where $\left\{\alpha_{n}\right\} \subset(0,1]$ and $\left\{e_{n}\right\}$ is an error sequence in $H$ satisfying conditions $(R 3)$ and (R4). Then $\left\{x_{n}\right\}$ strongly converges to $x^{*} \in F(S) \cap F(T)$, where $x^{*}=P_{F(S) \cap F(T)}(I-A+\gamma f) x^{*}$ is a solution of the following variational inequality:

$$
\left\langle(\gamma f-A) x^{*}, p-x^{*}\right\rangle \leq 0, \quad \forall p \in F(S) \cap F(T) .
$$

\footnotetext{
Author details

${ }^{1}$ Dipartimento di Matematica, Universita della Calabria, Arcavacata di Rende (Cs) 87036, Italy ${ }^{2}$ Department of Mathematics, Banaras Hindu University, Varanasi 221005, India
}

\section{Authors' contributions}

All the authors contributed equally and significantly in writing this paper. All authors read and approved the final manuscript.

\section{Competing interests}

The authors declare that they have no competing interests.

Received: 17 December 2011 Accepted: 15 May 2012 Published: 15 May 2012

\section{References}

1. Browder, FE: Nonexpansive nonlinear operators in a Banach space. Proc Nat Acad Sci USA. 54, 1041-1044 (1965). doi:10.1073/pnas.54.4.1041

2. Browder, FE: Convergence of approximants to fixed points of nonexpansive nonlinear mappings in Banach spaces. Arch Rat Mech Anal. 24, 82-90 (1967)

3. Reich, S: Strong convergence theorems for resolvents of accretive operators in Banach spaces. J Math Anal Appl. 75, 287-292 (1980). doi:10.1016/0022-247X(80)90323-6

4. Halpern, B: Fixed points of nonexpansive maps. Bull Am Math Soc. 73, 957-961 (1967). doi:10.1090/S0002-9904-196711864-0

5. Lions, P-L: Approximation de points fixes de contractions. CR Acad Sci Paris Ser A-B. 284, 1357-1359 (1977)

6. Wittmann, R: Approximation of fixed points of nonexpansive mappings. Arch Math. 58, 486-491 (1992). doi:10.1007/ BF01190119

7. Deutsch, F, Yamada, I: Minimizing certain convex functions over the intersection of the fixed point sets of nonexpansive mappings. Numer Funct Anal Optim. 19, 33-56 (1998)

8. Li, S, Li, L, Su, Y: General iterative methods for a one-parameter nonexpansive semigroup in Hilbert space. Nonlinear Anal. 70, 3065-3071 (2009). doi:10.1016/j.na.2008.04.007

9. Marino, G, Colao, V, Qin, X, Kang, SM: Strong convergence of the modified Mann iterative method for strict pseudocontractions. Comput Math Appl. 57, 455-465 (2009). doi:10.1016/j.camwa.2008.10.073

10. Marino, G, Xu, HK: A general iterative method for nonexpansive mappings in Hilbert spaces. J Math Anal Appl. 318, 43-52 (2006). doi:10.1016/j.jmaa.2005.05.028

11. Xu, HK: An iterative approach to quadratic optimization. J Optim Theory Appl. 116, 659-678 (2003). doi:10.1023/ A:1023073621589

12. Xu, HK: Iterative algorithms for nonlinear operators. J Lond Math Soc. 66, 240-256 (2002). doi:10.1112/ S0024610702003332 
13. Yamada, I, Ogura, N, Yamashita, Y, Sakaniwa, K: Quadratic approximation of fixed points of nonexpansive mappings in Hilbert spaces. Numer Funct Anal Optim. 19, 165-190 (1998). doi:10.1080/01630569808816822

14. Plubtieng, S, Punpaeng, R: Fixed point solutions of variational inequalities for nonexpansive semigroups in Hilbert spaces. Math Comput Model. 48, 279-286 (2008). doi:10.1016/j.mcm.2007.10.002

15. Cianciaruso, F, Marino, G, Muglia, L: Iterative methods for equilibrium and fixed point problems for nonexpansive semigroups in Hilbert spaces. J Optim Theory Appl. 146(2), 491-509 (2010). doi:10.1007/s10957-009-9628-y

16. Shioji, N, Takahashi, W: Strong convergence theorems for asymptotically nonexpansive mappings in Hilbert spaces. Nonlinear Anal. 34, 87-99 (1998). doi:10.1016/S0362-546X(97)00682-2

17. Shimizu, T, Takahashi, $\mathrm{W}$ : Strong convergence to common fixed points of families of nonexpansive mappings. J Math Anal Appl. 211, 71-83 (1997). doi:10.1006/jmaa.1997.5398

18. Agarwal, RP, O'Regan, D, Sahu, DR: Fixed point theory for Lipschitzian-type mappings with applications, Series. In Topological Fixed Point Theory and Its Applications, vol. 6,Springer, New York (2009)

19. Geobel, K, Kirk, WA: Topics in metric fixed point theory. In Cambridge Stud Adv Math, vol. 28,Cambridge Univ. Press, Cambridge (1990)

20. Aleyner, A, Reich, S: An explicit construction of sunny nonexpansive retractions in Banach spaces. Fixed Point Theory and Applications. 3, 295-305 (2005)

21. Edelstein, M, O'Brien, RC: Nonexpansive mappings, asymptotic regularity and successive approximations. J Lond Math Soc. 3, 547-554 (1978)

22. Takahashi, W: Nonlinear functional analysis, Fixed point theory and its applications. Yokohama Publishers, Yokohama (2000)

23. Martinet, B: Regularisation dinequations variationnelles par approximations successives. Rev FranMcaise Inf Recherche Operationnelle. 4, 154-158 (1970)

24. Rockafellar, RT: Monotone operators and the proximal point algorithm. SIAM J Control Optim. 14, 877-898 (1976). doi:10.1137/0314056

25. Sahu, DR, Yao, JC: The prox-Tikhonov regularization method for the proximal point algorithm in Banach spaces. J Glob Optim. 51, 641-655 (2011). doi:10.1007/s10898-011-9647-8

26. Kamimura, S, Takahashi, W: Approximating solutions of maximal monotone operators in Hilbert space. J Approx Theory 106, 226-240 (2000). doi:10.1006/jath.2000.3493

27. Blum, E, Oettli, W: From optimization and variational inequalities to equilibrium problems. Math Stud. 63, 123-145 (1994)

28. Oettli, W: A remark on vector-valued equilibria and generalized monotonicity. Acta Math Vietnam. 22, 215-221 (1997)

29. Combettes, PL, Hirstoaga, SA: Equilibrium programming in Hilbert spaces. J Nonlinear Convex Anal. 6(1), 117-136 (2005)

30. Iusem, AN, Sosa, W: Iterative algorithms for equilibrium problems. Optimization. 52(3), 301-316 (2003). doi:10.1080/ 0233193031000120039

31. Song, Y, Kang, Jl, Cho, YJ: On iterations methods for zeros of accretive operators in Banach spaces. Appl Math Comput. 216, 1007-1017 (2010). doi:10.1016/j.amc.2010.01.124

doi:10.1186/1687-1812-2012-83

Cite this article as: Colao et al:: A general inexact iterative method for monotone operators, equilibrium problems and fixed point problems of semigroups in Hilbert spaces. Fixed Point Theory and Applications 2012 2012:83.

\section{Submit your manuscript to a SpringerOpen ${ }^{\circ}$ journal and benefit from:}

Convenient online submission

- Rigorous peer review

- Immediate publication on acceptance

- Open access: articles freely available online

- High visibility within the field

- Retaining the copyright to your article

Submit your next manuscript at $\gg$ springeropen.com 